This item was submitted to Loughborough's Research Repository by the author.

Items in Figshare are protected by copyright, with all rights reserved, unless otherwise indicated.

\title{
Yarn twist measurement using digital imaging
}

PLEASE CITE THE PUBLISHED VERSION

http://dx.doi.org/10.1080/00405000802263476

PUBLISHER

Taylor and Francis / @ The Textile Institute

VERSION

AM (Accepted Manuscript)

LICENCE

CC BY-NC-ND 4.0

REPOSITORY RECORD

Ozkaya, Yasar A., Memis Acar, and Michael R. Jackson. 2019. "Yarn Twist Measurement Using Digital Imaging". figshare. https://hdl.handle.net/2134/13356. 
This item was submitted to Loughborough's Institutional Repository (https://dspace.lboro.ac.uk/) by the author and is made available under the following Creative Commons Licence conditions.

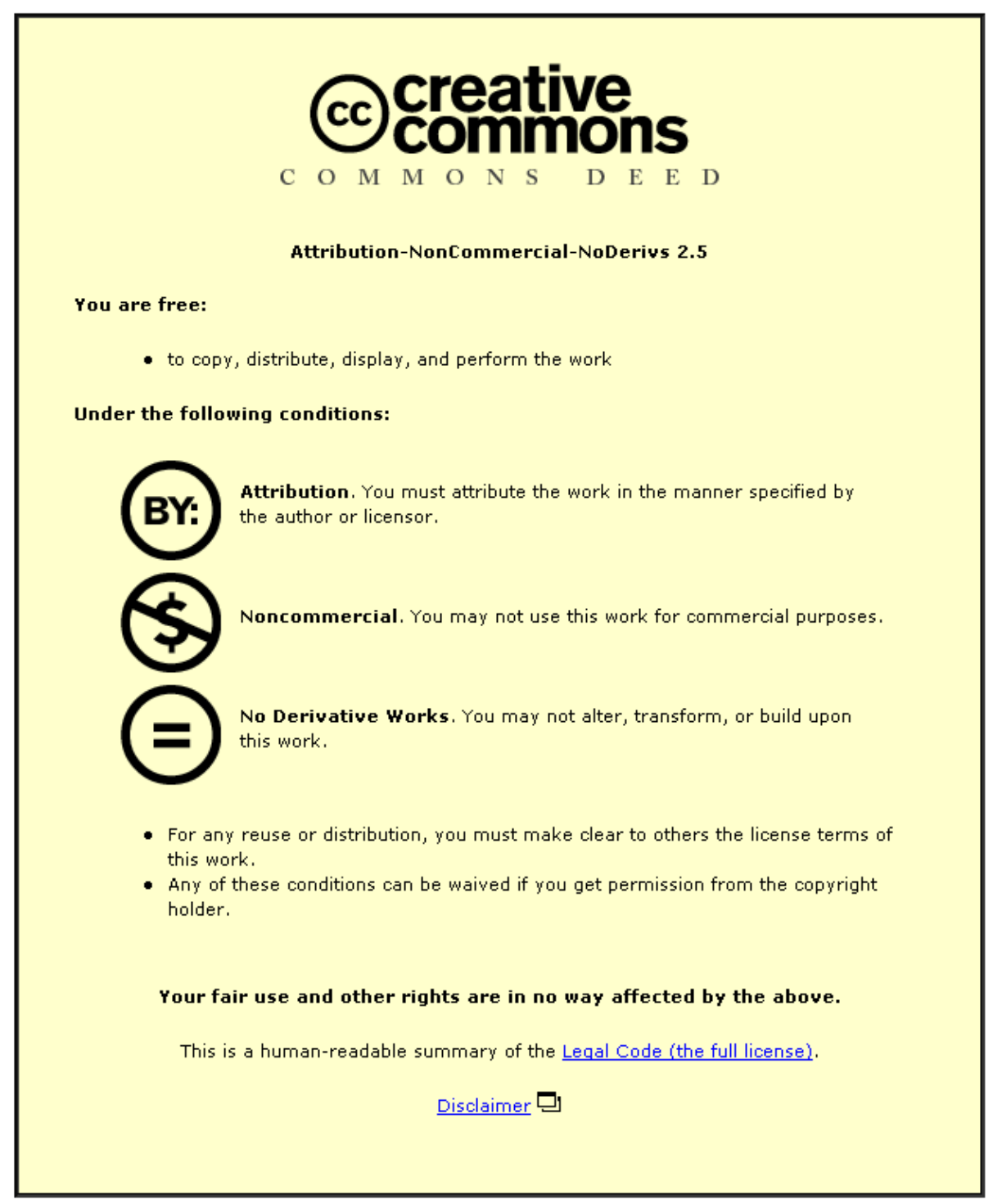

For the full text of this licence, please go to: http://creativecommons.org/licenses/by-nc-nd/2.5/ 


\title{
Yarn twist measurement using digital imaging
}

\author{
Y.A. Ozkaya, M. Acar* and M.R. Jackson \\ School of Mechanical and Manufacturing Engineering, Loughborough University, Loughborough, Leicestershire, UK
}

(Received 21 February 2008; final version received 9 June 2008)

\begin{abstract}
Digital image analysis techniques in the spatial and frequency domains for twist measurement of yarns are described. A spatial technique is developed to extract the twist angle through the analysis of the yarn core image. Then, a Fourier transformation technique is applied to yarn images to measure the orientation of the fibre on the yarn surface. Finally, a hybrid method that incorporates frequency domain filtering prior to spatial analysis is proposed. The trials show that spatial analysis is a fast method and can successfully predict the twist in the yarn. Fourier transformation technique is quite sensitive to the protruding fibres obstructing the yarn surface, which may result in measurements having high variations. For yarns having little amount of hairs protruding from the core, the results agreed reasonably well with actual twist levels. Frequency domain filtering in conjunction with the spatial analysis of the yarn surface is found to be superior in terms of accuracy. The twist values calculated using the more reliable diameter measurements with back-lit images together with twist angles from the front-lit images are found to be more accurate when compared with the actual values.
\end{abstract}

Keywords: yarn twist; digital imaging; twist angle; yarn diameter

\section{Introduction}

Twist is an important yarn parameter that affects the yarn characteristics such as strength, handle and appearance. The twist variation in a yarn creates irregular patterns on the fabric due to different dye absorption levels because low twisted regions absorb more dye compared with high twisted regions - the major reason for 'barre effect' in fabrics (Anbarasan, Upadhayaya, \& Balasubramaniam, 2003). Conventionally, yarn twist is measured by removing all the twists from a certain length of a yarn and then by retwisting to the same twist as proposed by Bellinson (1940). This method was initially applied by the aid of a pendulum in conjunction with a photronic cell. Later, it was semiautomated by the inclusion of a motor-driven twist counter. Although currently being the only accepted way to measure yarn twist, it is a time consuming and destructive method, which can only be performed off-line. Furthermore, the twist is measured on short lengths of yarn samples with this method, for example $50 \mathrm{~cm}$, which is obviously not sufficient to see high-frequency twist variations and hence not suitable to estimate the associated appearance problems on fabrics.

Relationship among specific volume, yarn count and twist of spun yarns was investigated by Hearle and Merchant (1963) whereas Sust and Barella (1964) studied twist, diameter and unevenness interrelations. Their findings paved the way to the estimation of yarn characteristics such as linear density and mass variation of yarns, which makes twist measurement an exciting part of vision-based yarn testing.

Recently, some computer vision techniques have been introduced to measure the yarn diameter, twist angle and hairiness in a non-destructive fashion (Cybulska, 1999; Vas, Halasz, Takacs, Eoerdoegh, \& Szasz, 1994). High-speed computer vision and digital analysis techniques were successfully applied to textured yarn interlace (nip) frequency measurements (Millman, Acar, \& Jackson, 2001), yarn core-hair separation to measure yarn diameter (Ozkaya, Acar, \& Jackson, 2005), the definition of hair density profile (Ozkaya, Acar, \& Jackson, 2007) and yarn hairiness measurement simulating conventional photosensor-based technique (Ozkaya, Acar, \& Jackson, 2008).

An application of image analysis technique was reported by Cork, Cooke, and Wild (1996) to measure the mean twist level and variation in twist of woven woollen fabrics in archaeological textiles. Ozkaya, Acar, and Jackson (2003) presented preliminary work on yarn twist measurement using a Fourier transformation (FT) technique, which has led to the development of methods for yarn twist measurement reported in this paper.

\section{Twist and twist angle}

Figure 1a shows the ideal geometry of a yarn having a diameter ' $d$ '. Because of twist, a surface fibre makes a helix angle $\theta$ with the core axis and completes one full rotation in $\mathrm{L}$ units parallel to the core axis, that is, there is 


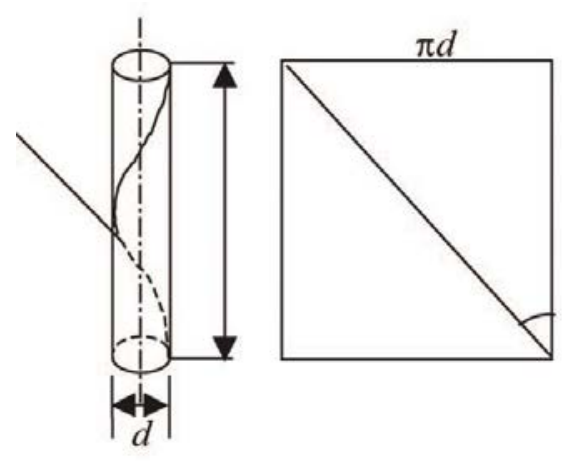

(a)

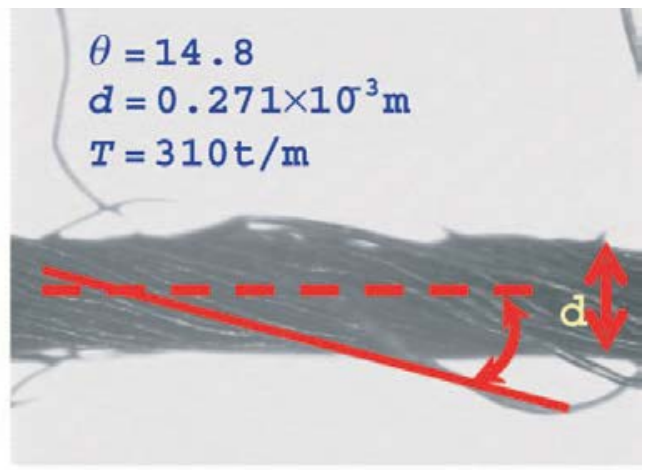

(b)

Figure 1. (a) An ideal yarn diagram showing the relation between twist angle and twist and (b) calculation of twist from a yarn image.

one rotation inserted into yarn every L units. The threedimensional structure can be unfolded into a right-angle triangle as shown in Figure 1a. From this triangle, the twist in unit length can be calculated using the following equation:

$$
T=\frac{\tan (\theta)}{\pi d}
$$

where the diameter $d$ is in meters, $T$ will give the twist in turns/meter. Figure 1b shows calculation of twist per unit length on a real yarn image.

Another parameter of yarn twist is the twist direction, which can be either ' $S$ ' or ' $Z$ ' as depicted in Figure 2.

\section{Core extraction}

For twist measurement, a Hitachi HV C-10 CCD area scan camera attached to a Meiji Techno microscope is used. The images are transferred to the computer using a Viper Quad analogue frame grabber at a frame size of $640 \times 480$. A ring light is employed to illuminate the surface of the yarns from the front. Varying magnifications between 2.5:1 and 4:1 are used depending on the fibre fineness of the yarns being tested. A technique reported by Ozkaya et al. (2005) has been used to determine the diameter of the fibres.

The image-processing task for measuring the twist involves two stages. In the first stage, the core region of the yarn is extracted from the image to perform two essential tasks - the first one being the determination of diameter and
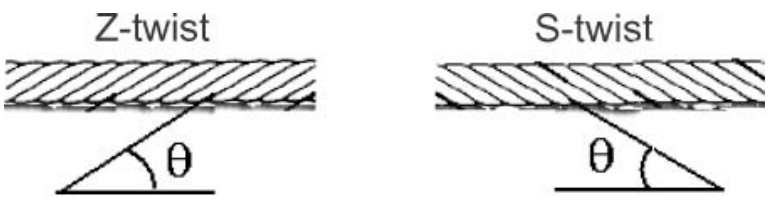

Figure 2. Twist directions. the second is the definition of the area of interest in which the twist angles will be searched.

Figure 3 shows a typical image used for twist measurement. Because the surface of the yarn is not uniformly bright, separating the core from surrounding hairs and the background using a thresholding or a tracking method would be problematic. Hence, an algorithm similar to the one used for processing dark field images (Millman et al., 2001) is developed.

Initially, 512-pixel-wide image portions that correspond to between 1.35 and $2.25 \mathrm{~mm}$ of the yarn section depending on the magnification are extracted from the original image. For each portion, the horizontal projections are calculated. The reason for partitioning the images into 512 pixel-wide segments is for convenience for the frequency domain analysis. Once the horizontal projection is obtained, the boundaries of the core are selected as the region falling above the value defined as one third from the maximum to the average value of the profile as shown in Figure 4.

Initially, two different methods are developed to determine the surface orientation angles. The first one is based on

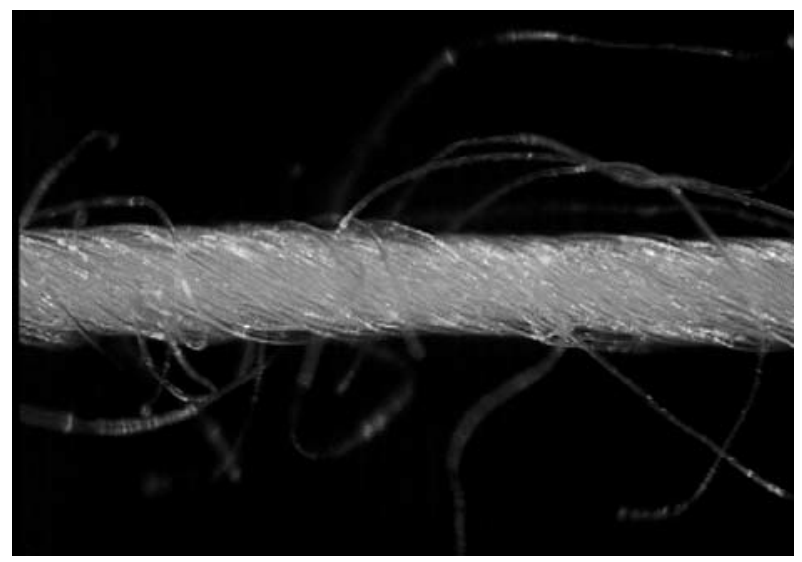

Figure 3. A typical yarn image for twist measurement. 


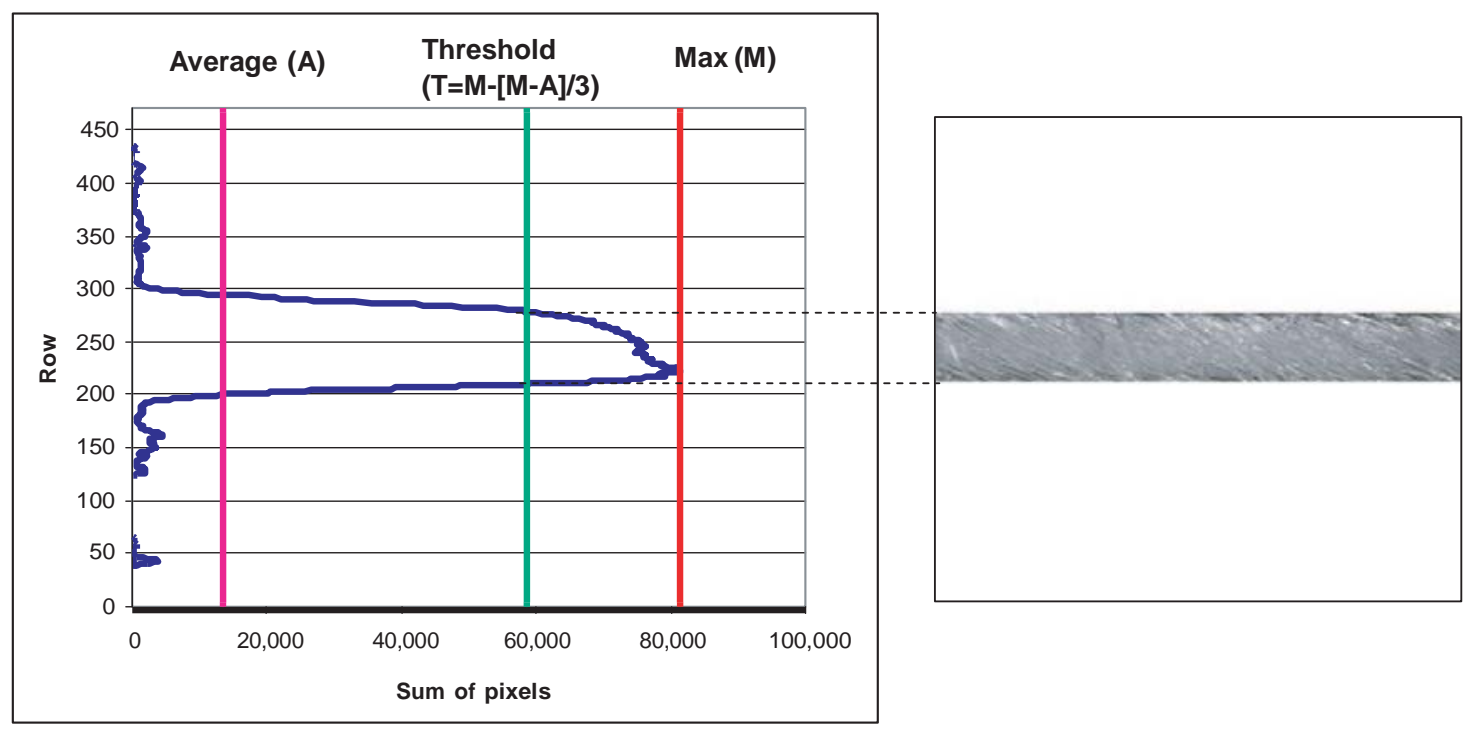

Figure 4. Left: the horizontal projection of the image in Figure 3 showing the threshold line and right: extracted core region.

spatial analysis whereas the second uses frequency domain analysis. Then, a hybrid method that combines frequency domain filtering with spatial analysis is also investigated.

\section{Spatial analysis method to extract the twist angle}

This process involves extraction of the twist angles through the analysis of the yarn core surface. An example of this process is illustrated in Figure 5. The algorithm starts by applying a $3 \times 3$ ideal low pass filter to the extracted core image to decrease the effect of noise while retaining the twist contours. To extract the relatively bright surface fibres, a Sobel filter is applied to this image at a slope threshold value of 12 and then by skeletonising, these fibres are reduced to 1-pixel-wide contours. To extract the contours resembling a line from this image, first of all, the contours are converted to a polyline structure based on 8-connected neighbourhood of pixels. Then, line segments are produced from these polylines by checking whether any vertices of the polyline endpoints are more than 4 pixels away from the line connecting its two endpoints; if not, polyline endpoints are considered as line segment endpoints; otherwise, the polyline is split at the vertex farthest from the line, and the algorithm is applied recursively to the two resulting polylines. Finally, lines found on the skeleton are merged if the angles are within a $2^{\circ}$ tolerance and the endpoints of matching ends are within 5 pixels distance. The lines shorter than 15 pixels are filtered out during this procedure. All these tasks are performed using built-in Wit 7.1 operators.

As a yarn may have either 'S' or ' $Z$ ' twist only, the slopes of the lines representing the twist must be in a certain range. The lines having angles between 10 and 80 that correspond to ' $\mathrm{S}$ ' twist and those having angles between 100 and 170 that correspond to ' $\mathrm{Z}$ ' twist are separated from each other, and the dominant line direction is accepted to be the twist direction of the yarn whereas a small number of lines in the opposite direction are omitted. Once finding the twist direction, the weighed average of the angles of the lines in the dominant direction is calculated by multiplying the angles with the lengths and then dividing it by the total length of the lines.

\section{Frequency domain analysis}

The frequency domain analysis to measure the twist angle involves the idea that the fibres on the surface have similar spatial characteristics (thickness and brightness) that lead to similar frequency components, and because the fibres on the yarn surface are aligned in similar directions, the fibre frequencies might be expected to concentrate in a region that is directly related to the twist angle. When imaging the yarn surface, there is a high possibility that the surface will be obstructed by hairs protruding from the core. However, these hairs will be significantly defocused compared to the fibres on the surface. In this case, the spectra of these fibres will mainly consist of low frequencies.

The algorithm developed for frequency domain analysis of the yarn surface images starts by overlaying the 512-pixel-wide core portion onto a $512 \times 512$ image to enable a square fast Fourier transform (FFT). Figure 6 shows the overlaid core image (a) and its FT (magnitude) on a logarithmic scale (b). Two very interesting frequency components are observed in the Fourier domain: first one corresponding to a sinusoidal aligned to $45^{\circ}$ in the image with a frequency of 0.5 cycles/pixel and the other aligned to $71^{\circ}$ with a frequency of 0.38 cycles/pixel. It later became clear 


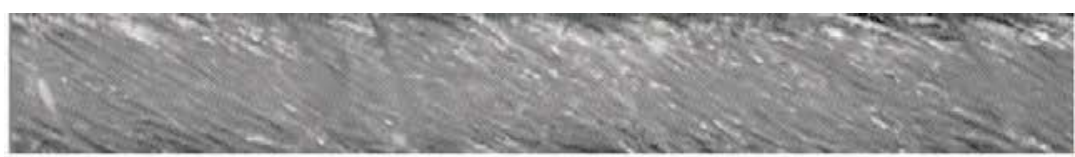

Original image

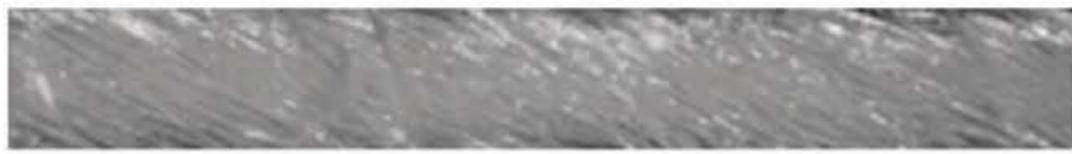

After low pass filtering

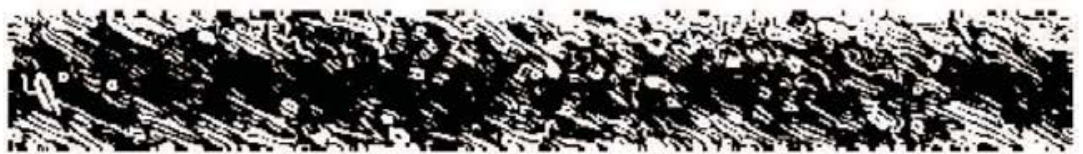

After applying a Sobel filter and thresholding

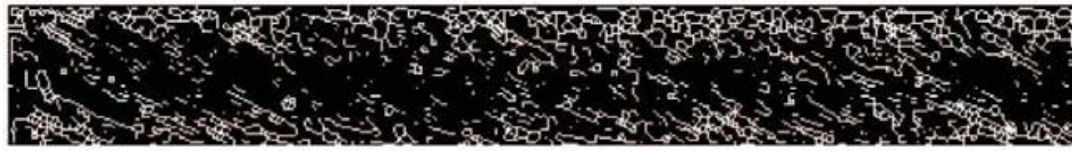

After skeletonising

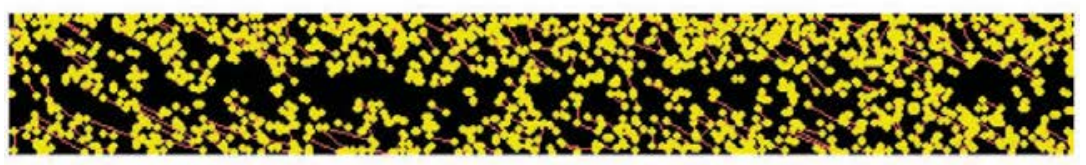

Lines extracted from skeleton contours

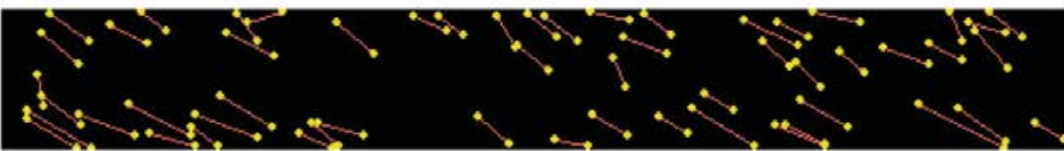

After merging close lines and filtering out the short ones

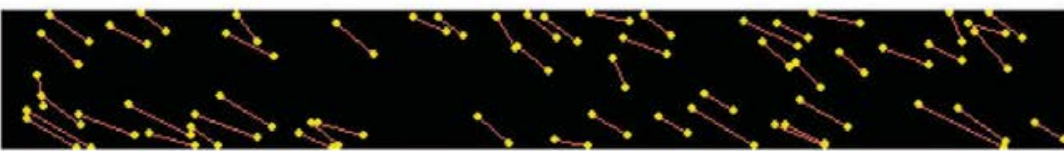

Lines in the dominant direction (S) are extracted

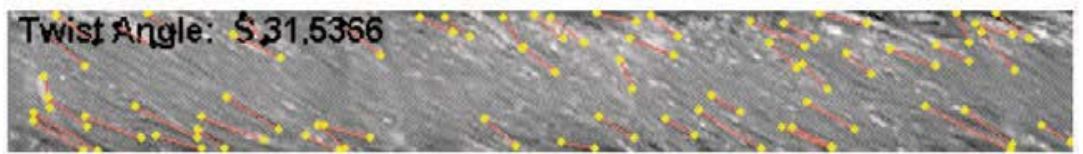

Output of the algorithm showing the weighed twist angle average

Figure 5. Steps of the algorithm to determine the twist angle.

that these sinusoidal noises were added to the image due to a problem with the analogue frame grabber used in the experiments.

The frequencies appearing at $v \cong 0$ (vicinity of horizontal axis) mainly arise from the image edges (left and right) and $u \cong 0$ (vertical axis) mainly due to the sharp crossing at the bottom edge of the core in the image. The fibres lying vertically and horizontally would also contribute to the frequencies along $u$ and $v$ axis. Because these frequencies are not of interest, they can be eliminated simply by removing the pixels whose coordinates fall in the areas where $|u| \leq$ 2 and $|v| \leq 2$. 


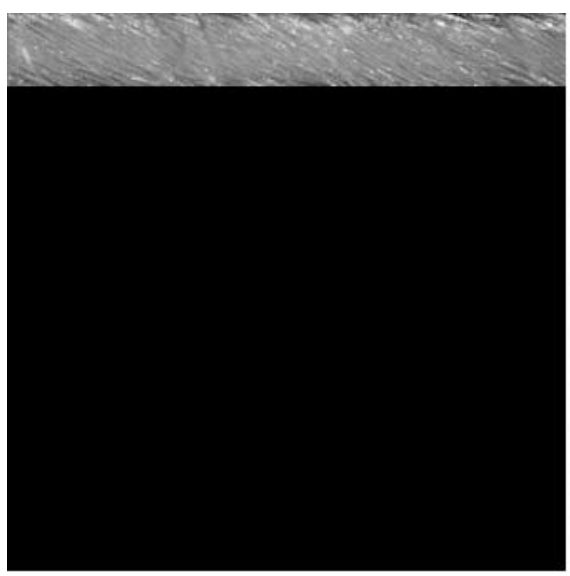

(a)

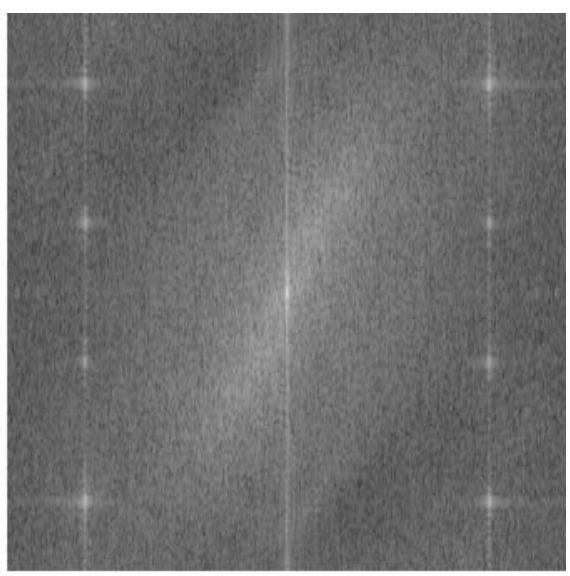

(b)

Figure 6. (a) Image of the yarn core and (b) magnitude of FT on logarithmic scale.

If the fibre has a width of $n$ pixels in an $N \times N$ image, the following band pass filter can be applied in the frequency domain:

$$
\frac{N}{2 n}<\overline{u^{2}+v^{2}}<\frac{N}{n}
$$

This filtering will get rid of high frequencies from different sources such as the sinusoidal noise caused by the frame grabber without altering the information from the fibres, as well as the low frequency components that mainly arise from yarn core and out of focus protruding fibres.

Furthermore, frequencies can also be filtered according to the twist direction of the yarn. Figure 7 shows the positions of frequencies corresponding to surface fibres making a twist angle $\theta$ in either $\mathrm{S}$ or $\mathrm{Z}$ direction. If the twist direction of a yarn sample is known, then the frequencies that would arise from the opposite twist direction can be omitted (e.g. zones 2 and 4 can be filtered out if the yarn has

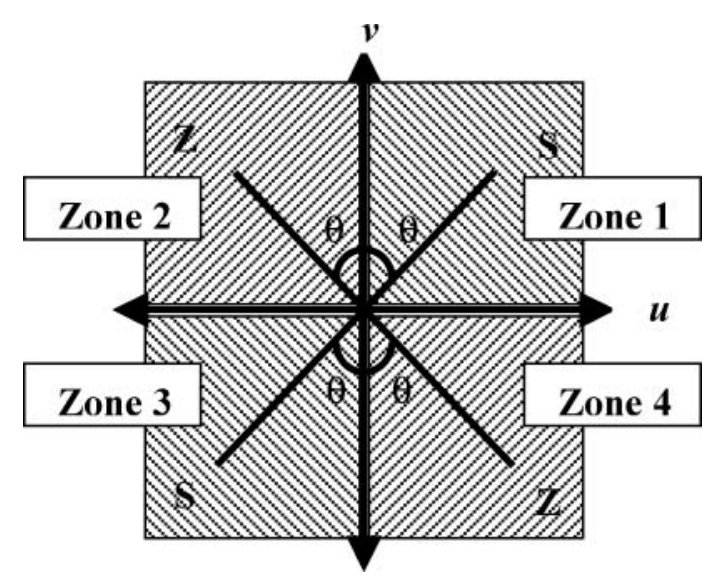

Figure 7. Frequency components of $\mathrm{S}$ and $\mathrm{Z}$ twists.
$\mathrm{S}$ twist). This filtering helps to eliminate the surface hairs lying in opposite directions to the twist angle.

Figure 8 shows the magnitude component of the spectrum after applying the aforementioned filters.

The final step of the algorithm is to find the dominant direction in the frequency domain. The first approach for this is to calculate the mean magnitude along each angular direction incremented by 1 from $0^{\circ}$ to $90^{\circ}$ within the zone corresponding to the twist direction (e.g. zone 1 for $\mathrm{S}$ twist). Figure 9 shows the mean magnitudes along each direction from $0^{\circ}$ to $90^{\circ}$. The maximum mean magnitude appears along $67^{\circ}$, which indicates an $\mathrm{S}$ twist of $23^{\circ}$.

Another approach to determine the twist angle is simply to find the angle that the $v$ axis makes with the line between

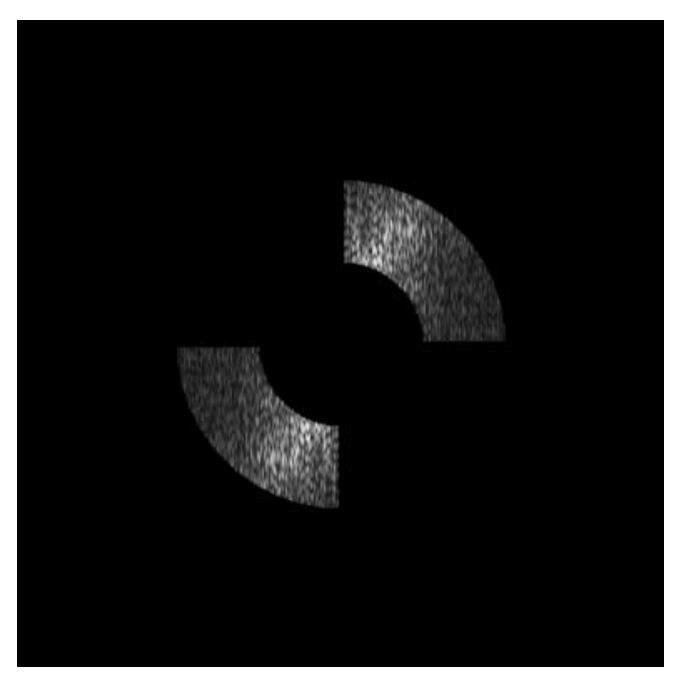

Figure $\rho$. Filtered FT (magnitude), frequencies falling in range $65<\overline{u^{2}+v^{2}}<130$ within zones 1 and 3 are extracted from the original spectrum. 


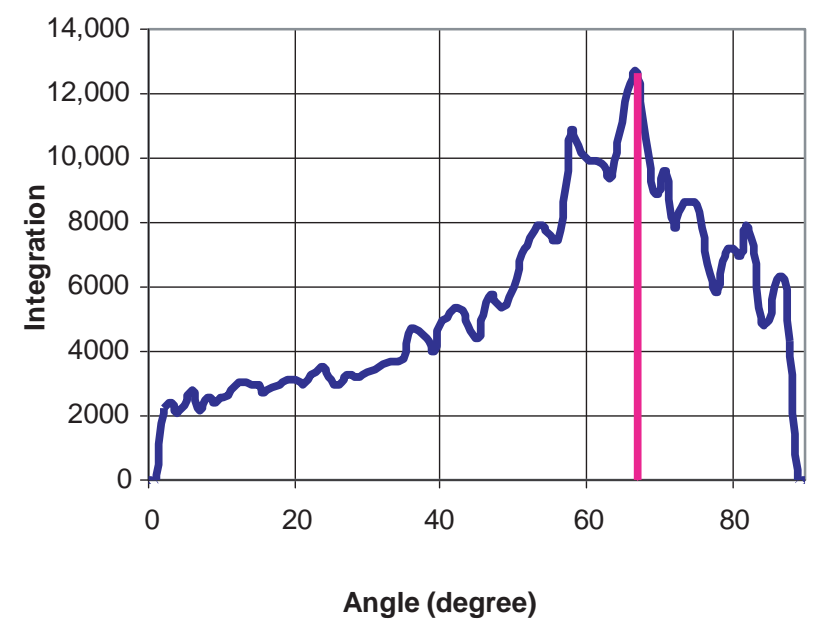

Figure 9. Mean magnitudes along varying angles.

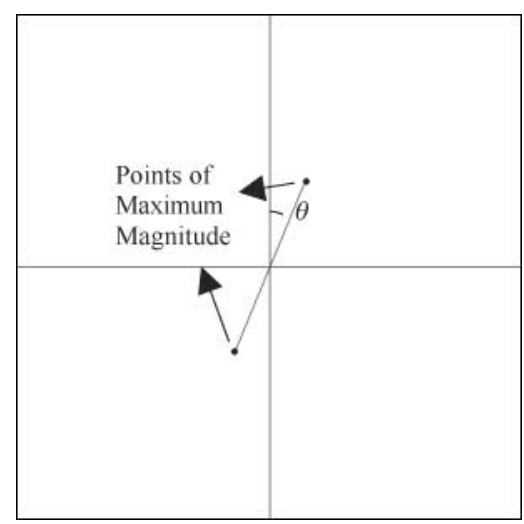

Figure 10. Determination of twist angle from the frequency having maximum magnitude.

the pixels having the maximum magnitude in the spectrum as depicted in Figure 10. The calculated twist angle this time is $22.38^{\circ}$.

One can expect the twist angles calculated using these two different methods to be in good agreement with each other. However, for many images especially for those having a complicated surface structure, they did not correlate well and in such cases both measurements often gave inaccurate results. For this reason, the twist angle is taken to be the average of the twist angles calculated from these two different methods if they are within $2^{\circ}$ proximity; otherwise, both measurements are discarded.

\section{Hybrid method: frequency domain filtering and spatial analysis}

After working on spatial and frequency domain analysis techniques, it became clear that a hybrid method that in-

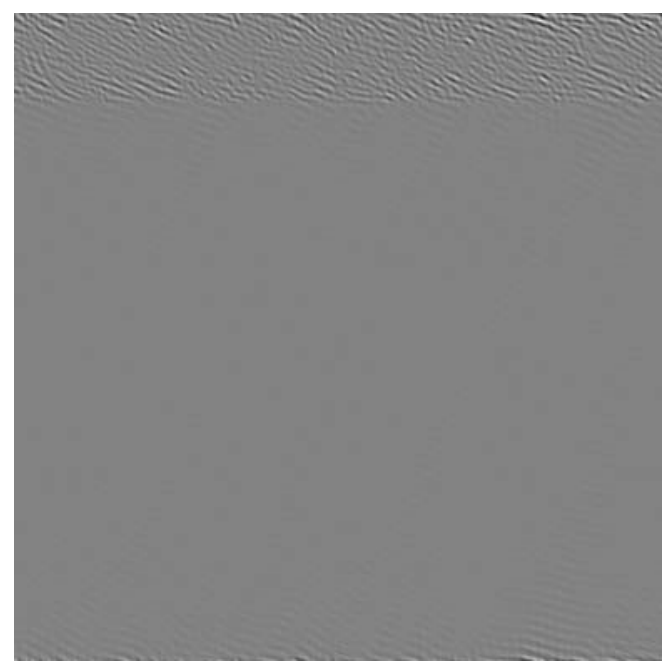

Figure 11. Inverse transform of the filtered spectrum.

corporates frequency domain filtering followed by spatial analysis would be an alternative method for yarn surface inspection.

Figure 11 shows the inverse transform of the filtered spectrum. When a threshold at 0 is applied to the inverse transform, the improvement in the image compared with the original image can be seen in Figure 12.

From this point, the spatial analysis algorithm can be applied to the image Figure 12b. However, in this case both the low pass and the Sobel edge detection filters are not required, and the algorithm can directly start from the skeletonising step. Figure 13 shows the output of this algorithm. It can be seen that the contours extracted in this case are more significant.

This technique worked very well even on yarn images with poor surface detail. Figure 14a shows such an image processed using both the spatial analysis algorithm and the hybrid method. It can be seen that the spatial analysis algorithm fails to detect the details with little contrast leading to inaccurate measurements. On the other hand, when the frequency domain filtering is applied, these details are significantly highlighted. Through a careful comparison, Figures 14a and d, it can be concluded that the hybrid method is very successful in determining the surface fibre contours.

The main disadvantage of the hybrid algorithm is the time required to process the images because the method requires two FFT operations - one in forward and the other in reverse directions. To speed up the algorithm, $64 \times 64$ windows are used rather than $512 \times 512$ as shown in Figure 15a. This corresponds to a 12 times reduction in computation time. However, it should be noted that a $64 \times$ 64 frame size may not be suitable for frequency domain analysis due to increasing error arising from digitisation. 


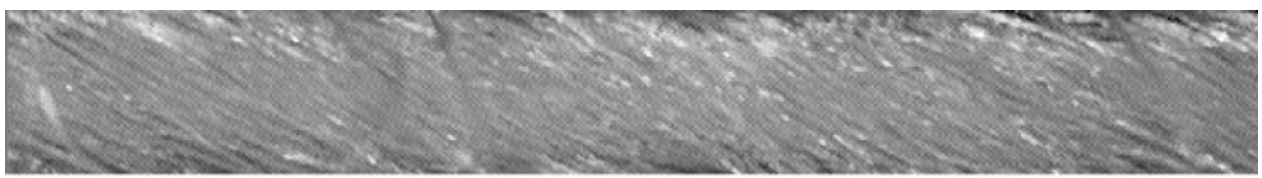

(a)

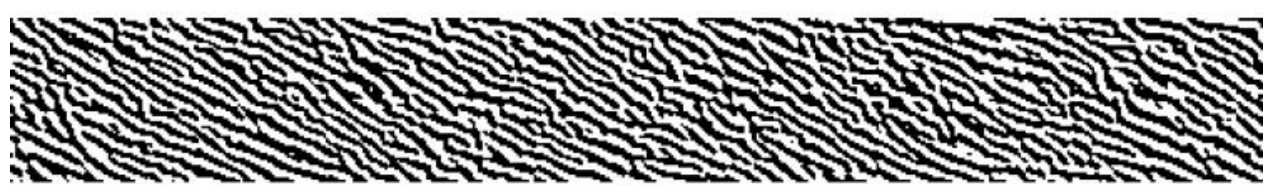

(b)

Figure 12. (a) Original core image and (b) inverse of filtered FT after thresholding at 0.

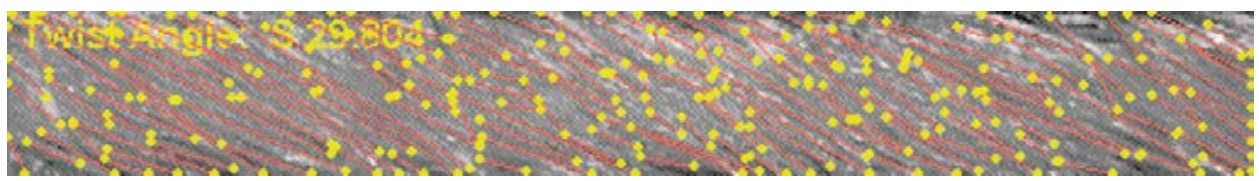

Figure 13. Output of the hybrid-processing algorithm.

\section{Results and discussion}

Twenty-eight different yarn samples are tested to investigate the accuracy of the algorithms. Around 60 images are taken from each sample, and these images are then analysed using all three methods described.

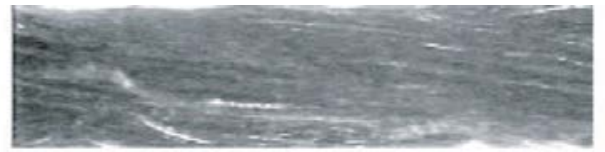

(a) Original core image (contrast is enhanced for visibility?

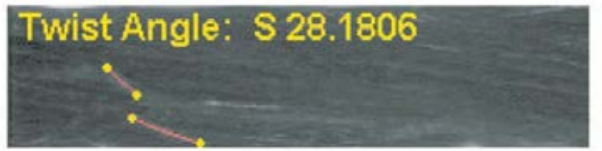

(b) Output of spatial analysis algorithm

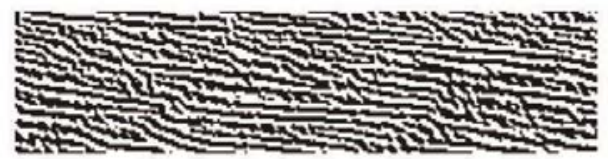

(c) Frequency domain filtered and thresholded image

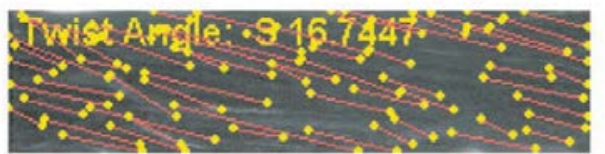

(d) Output of the hybrid method

Figure 14. Hybrid method applied on a yarn image with poor surface detail.
An important difference between the frequency domain and spatial domain analysis is that, in the frequency domain, the twist angle value is obtained from the most significant direction whereas for the spatial analysis method, a weighed average is calculated, which is more likely to be influenced by the surface hairs. Another important difference is the condition check that applies to the frequency domain analysis omitting images that do not exhibit consistent twist angles from maximum magnitude and the angular integration techniques. As a result of this condition check, the percentage of valid images varied between $25 \%$ and $50 \%$ for the frequency domain analysis whereas on the other hand, all images were taken into account for spatial analysis.

Figure 16 shows the twist measurements using the three methods and compares them with the actual twist values obtained from the manufacturing labels of the yarn packages. The frequency domain analysis method appears to exhibit much closer agreement with the actual values for most of the samples. The spatial analysis method on the other hand exhibits generally significantly higher twist values, whereas the hybrid method gives values falling between the values from the frequency domain and spatial domain analysis but still considerably above the actual values. The correlations with the actual values are quite low for all three methods (spatial, hybrid and frequency domain) with $R^{2}$ values of $0.42,0.45$ and 0.5 , respectively. The main reason for overestimation of the twist values by all three techniques is that the front-lit illumination method used in determining the yarn diameter is not as accurate as the back-lit illumination technique and that it underestimates the yarn diameter, which in turn gives higher twist per unit length values (Millman et al., 2001). 


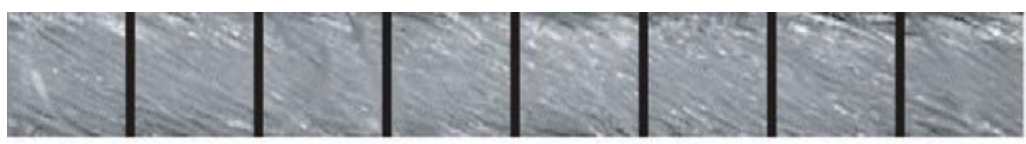

(a) Core image segmented into $64 \times 64$ portions

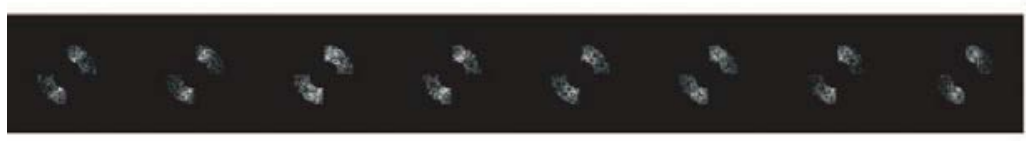

(b) Filtered spectrums of each individual portion

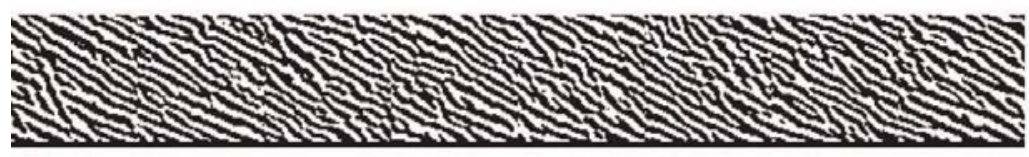

(c) After taking the inverse FFT, thresholding and merging the portions

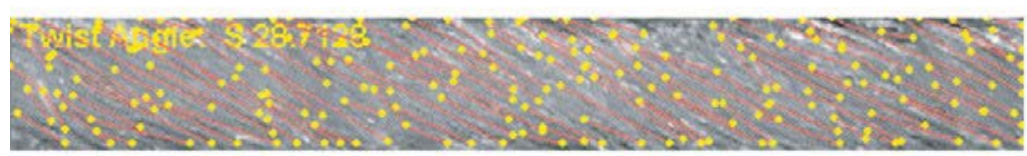

(d) The output of spatial analysis algorithm applied to the image in (c)

Figure 15. Application of the hybrid method using $64 \times 64$ image portions.
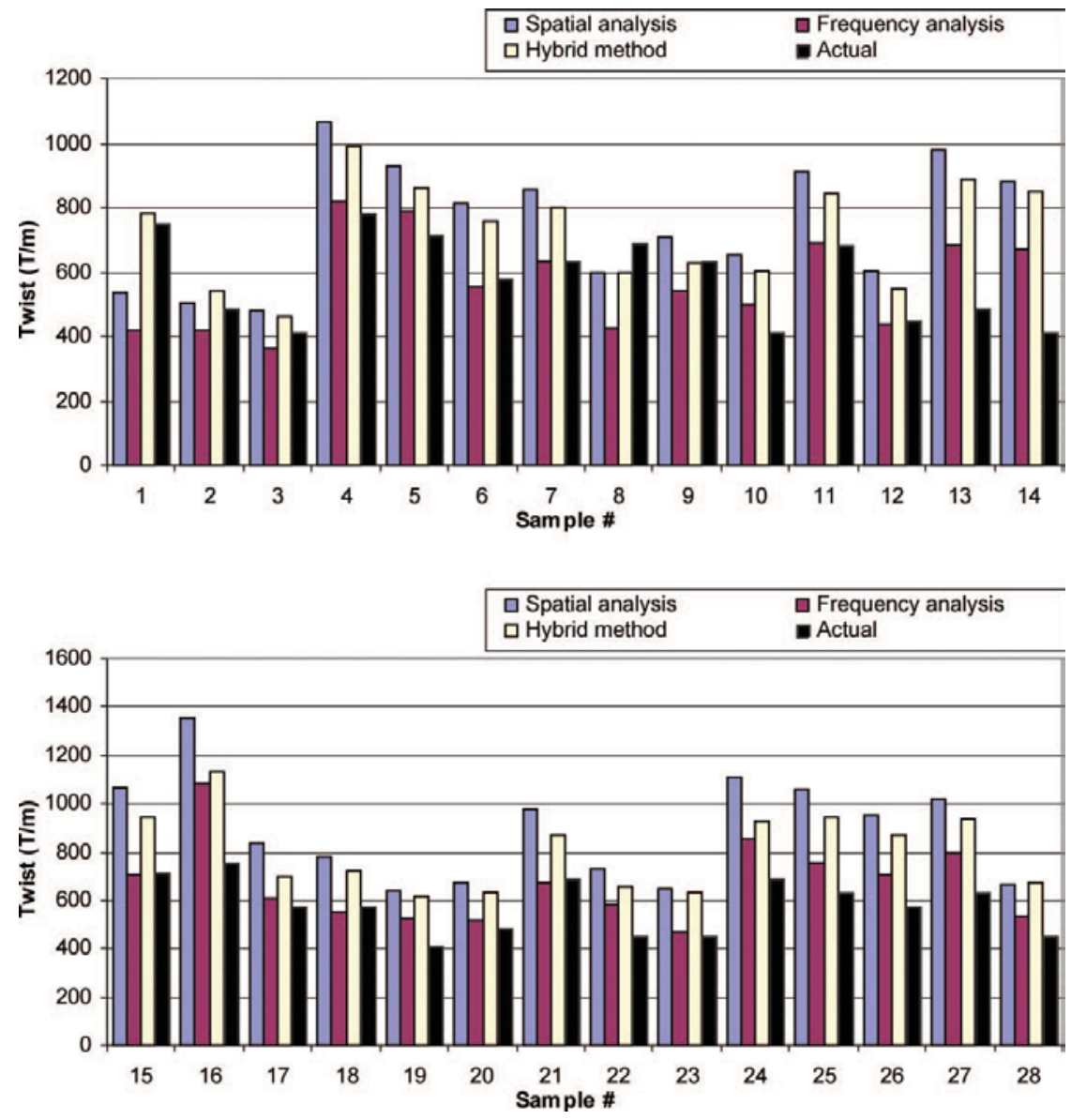

Figure 16. Comparison of actual twist values with the measurements from the three different algorithms for 28 yarn samples. 

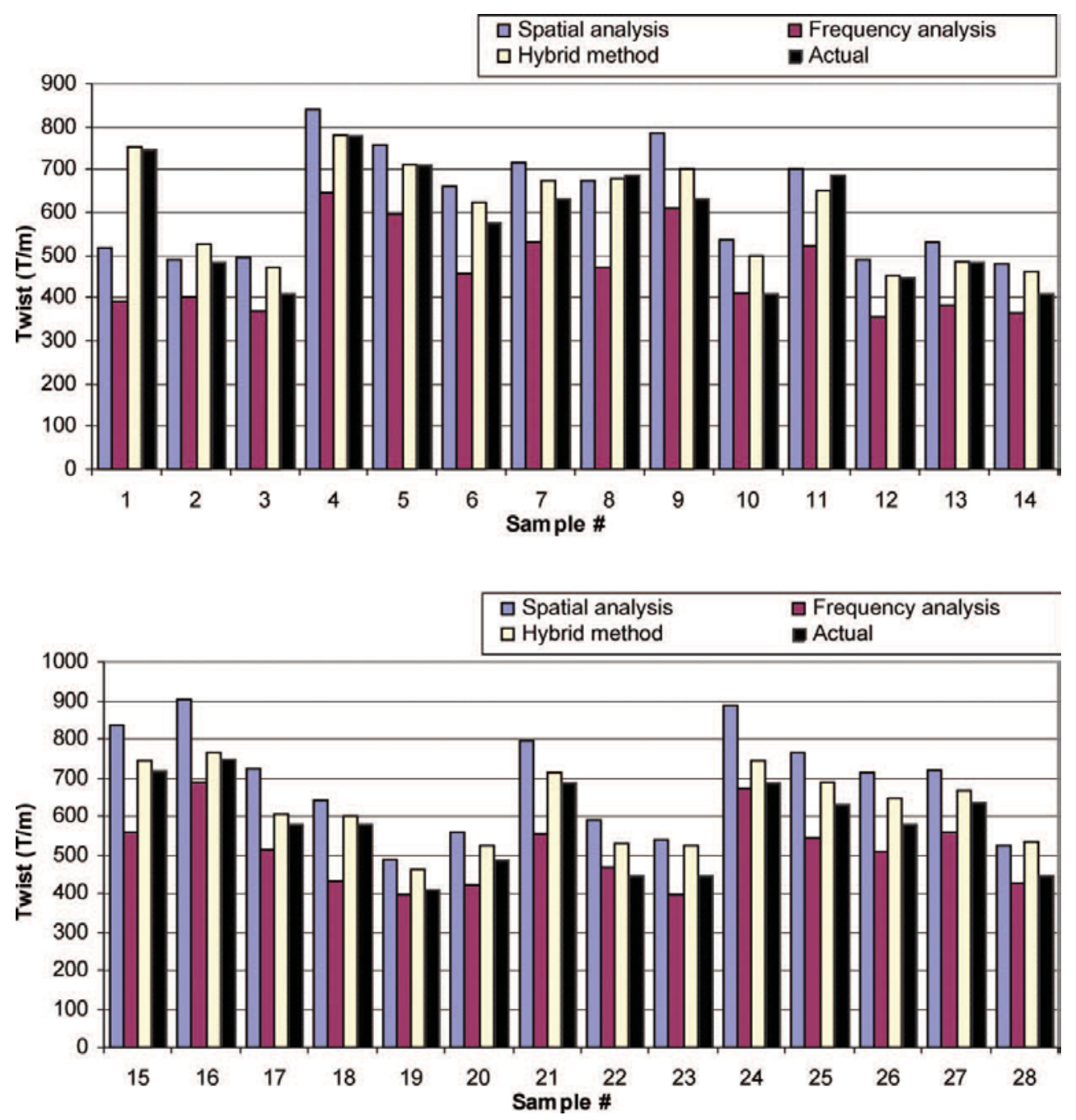

Figure 17. Comparison of actual twist values with those calculated by the three different algorithms and the mean diameters from back-lit images.

Figure 17 shows the analysis results obtained using the twist values calculated using the mean diameters from the back-lit images and mean twist angles from three different techniques and the actual twist values. In general, all three techniques demonstrate much more accurate results when diameter measurements with the back-lit yarn images are used. The hybrid method exhibits a very good agreement with the actual twist values, which gave a correlation coefficient of 0.94 . The spatial analysis and frequency analysis methods also exhibited reasonable correlations with the actual values with coefficients of 0.87 and 0.79 , respectively. These results strongly suggest that core extraction algorithm that uses the front-lit illumination should be revised to improve the accuracy of the twist measurement technique using the front-lit illumination. The best way to do this would probably be the use of a front- and back-lit hybrid yarn illumination because the diameter is better defined in back-lit images.
A comparison among three different algorithms in terms of time to process $512 \times 512$ images has been made. It is apparent that the time required to carry the fast Fourier transformation on $512 \times 512$ images is typically $4-5$ times slower than the other two techniques. The time required to process a 1-m long yarn section using frequency domain analysis could take several minutes. The speed of processing can be increased significantly by using a more advanced, faster CPU.

\section{Conclusions}

Three digital imaging techniques to compute yarn twist angle, namely the spatial analysis, frequency domain analysis and hybrid method, have been explained. It has been found that the spatial analysis method is the fastest but not the most accurate. The frequency components of a yarn image are seriously affected by fibres covering the yarn surface, 
which reduce the reliability of frequency domain analysis for most of the images. The hybrid method involving frequency domain filtering followed by spatial analysis is superior in terms of accuracy.

The twist measurements calculated using the diameter measurements from back-lit images in conjunction with the twist angles from the front-lit images are found to be more accurate for all three techniques. This is attributed to the diameter measurements from the back-lit yarn images, which are much more accurate than those determined using front-lit images.

\section{References}

Anbarasan, M., Upadhayaya, M.B., \& Balasubramaniam, V. (2003). Fibre and yarn property influences on fabric 'Barre' part-I, influence of yarn hairiness and twist. Premier1 Newsletters. Retrieved October 2003, from http://www. premier-1.com

Bellinson, H.R. (1940). Twist determination in single yarns. Textile Research Journal, 10(3), 120-125.

Cork, C.R., Cooke, W.D., \& Wild, J.P. (1996). The use of image analysis to determine yarn twist level in archaeological textiles. Archaeometry, 38(2), 337-345.

Cybulska, M. (1999). Assessing yarn structure with image analysis methods. Textile Research Journal, 69(5), 369-373.
Hearle, J.W.S., \& Merchant, V.B. (1963). Relations between specific volume, count, and twist of spun nylon yarns. Textile Research Journal, 33(6), 417-424.

Millman, M.P., Acar, M., \& Jackson, M.R. (2001). Computer vision for textured yarn interlace (nip) measurements at high speeds. Mechatronics, 11(8), 1025-1038.

Ozkaya, Y.A., Acar, M., \& Jackson, M.R. (2003). A Fourier transformation technique for yarn twist measurement. In R.M. Parkin, A. El-Habaibeh, \& M.R. Jackson (Eds.), ICOM 2003-International conference on mechatronics (pp. 303307). Loughborough, UK: Wiley.

Ozkaya, Y.A., Acar, M., \& Jackson, M.R. (2005). Digital image processing and illumination techniques for yarn characterization. Journal of Electronic Imaging, 14(2), 1-13.

Ozkaya, Y.A., Acar, M., \& Jackson, M.R. (2007). Hair density distribution profile to evaluate yarn hairiness and its application to fabric simulations. Journal of the Textile Institute, 98(6), 483-490.

Ozkaya, Y.A., Acar, M., \& Jackson, M.R. (2008). Simulation of photosensor-based hairiness measurement using digital image analysis. Journal of the Textile Institute, 99(2), 93100.

Sust, A., \& Barella, A. (1964). Twist, diameter and unevenness of yarns-a new approach. Journal of Textile Institute, 55, T1-T6.

Vas, L.M., Halasz, G., Takacs, M., Eoerdoegh, I., \& Szasz, K. (1994). Measurement of yarn diameter and twist angle with image processing system. Periodica Polytechnica Ser Mechanical Engineering, 38(4), 277-296. 